\title{
On turbulent entrainment and dissipation in dilute polymer solutions
}

\author{
A. Liberzon, ${ }^{1}$ M. Holzner, ${ }^{2}$ B. Lüthi, ${ }^{2}$ M. Guala, ${ }^{3}$ and W. Kinzelbach ${ }^{2}$ \\ ${ }^{1}$ School of Mechanical Engineering, Faculty of Engineering, Tel Aviv University, Tel Aviv 69978, Israel \\ ${ }^{2}$ Institute of Environmental Engineering, ETH Zurich, CH 8093 Zurich, Switzerland \\ ${ }^{3}$ Graduate Aeronautical Laboratories, California Institute of Technology, Pasadena, California 91125, USA
}

(Received 28 April 2008; accepted 29 January 2009; published online 26 March 2009)

\begin{abstract}
We present a comparative experimental study of a turbulent flow developing in clear water and dilute polymer solutions ( 25 and $50 \mathrm{wppm}$ polyethylene oxide). The flow is forced by a planar grid that oscillates vertically with stroke $S$ and frequency $f$ in a square container of initially still fluid. Two-component velocity fields are measured in a vertical plane passing through the center of the tank by using time resolved particle image velocimetry. After the forcing is initiated, a turbulent layer develops that is separated from the initially irrotational fluid by a sharp interface, the so-called turbulent/nonturbulent interface (TNTI). The turbulent region grows in time through entrainment of surrounding fluid until the fluid in the whole container is in turbulent motion. From the comparison of the experiments in clear water and polymer solutions we conclude: (i) Polymer additives modify the large scale shape of the TNTI. (ii) Both, in water and in the polymer solution the mean depth of the turbulent layer, $H(t)$, follows the theoretical prediction for Newtonian fluids $H(t) \propto \sqrt{K t}$, where $K \propto S^{2} f$ is the "grid action." (iii) We find a larger grid action for dilute polymer solutions than for water. As a consequence, the turbulent kinetic energy of the flow increases and the rate of energy input becomes higher. (iv) The entrainment rate $\beta=v_{e} / v_{\text {rms }}$ (where $v_{e}=d H / d t$ is the interface propagation velocity and $v_{\mathrm{rms}}$ is the root mean square of the vertical velocity) is lower for polymers $\left(\beta_{p} \approx 0.7\right)$ than for water $\left(\beta_{w} \approx 0.8\right)$. The measured values for $\beta$ are in good agreement with similarity arguments, from which we estimate that in our experiment about $28 \%$ of the input energy is dissipated by polymers. () 2009 American Institute of Physics. [DOI: 10.1063/1.3097006]
\end{abstract}

\section{INTRODUCTION}

Since the drag reduction phenomenon discovery by Toms, ${ }^{1}$ it is well known that turbulent flows can be strongly modified by very dilute solutions of flexible polymers, e.g., Ref. 2. There is a large literature body on the subject: see, for example, the review ${ }^{3}$ that lists more than 2500 entries. Since 1995 the number of contributions has increased considerably, see, for example, the recent publications of Refs. 4 and 5 for an updated list of references.

In spite of extensive research, the physical mechanisms underlying the effect of dilute polymers on turbulence remain poorly understood. Drag reduction is the most well known large scale phenomena, although there is a consensus that the direct action of the polymers is on the small scales of turbulent flows, e.g., Ref. 6 (and references therein). Simpler experiments have been devised in which the effects at large and small scales could be separated and the effect associated with the boundaries, i.e., mechanism of turbulence agitation, could be isolated from the effect in the turbulent bulk. There are several examples of turbulent flows of this kind such as the spreading of a turbulent cloud $^{7}$ or counter-rotating disk setups. ${ }^{8-10}$

The nature of the effect of dilute polymers on different turbulent free shear flows has been studied by several authors. There are works ${ }^{11-16}$ and more recent Ref. 17 that show how in grid turbulence the turbulent kinetic energy decays slower in dilute polymer solutions than in clear water. Support of the observation of the modified turbulent flow far from the boundaries was later given by the experiments with the so-called French washing machine facility, ${ }^{8}$ and in a similar apparatus. ${ }^{10,18}$ In experiments of turbulent cloud spreading, ${ }^{7,19}$ the dispersion was found to be slower in dilute polymer solutions, which points to reduced entrainment rates in these fluids.

Oscillating grid-stirred flow chambers have long been used in studies of turbulent mixing and transport processes. ${ }^{20,21}$ The flow produced by an oscillating grid is the result of interactions between the individual jets and wakes created by the motion of the grid bars. At sufficient distance from the grid, these jets and wakes interact and break into turbulence that propagates away from the grid. Ideally, no mean flow exists and the turbulence is considered nearly isotropic and homogeneous in planes parallel to the driving grid. In this work we study the turbulent flow developing below an oscillating grid, installed at the top of a water tank. Our main question is how polymer additives affect the evolution of the turbulent/nonturbulent interface (TNTI) and the turbulent entrainment process. We examine the roles played by the large and small scales of the flow and their interactions (with and without polymers) in the context of energy input and dissipation.

In self-preserving turbulent flows (e.g., jets, wakes, and boundary layers), the interface separating the turbulence from the irrotational fluid advances into the ambient space at rates that are governed by the large scale properties of the flow. All these flows are known to spread according to power laws ${ }^{22}$ and the propagation velocity $v_{e}$ is proportional to the velocity scale of the energy-containing turbulence behind the 


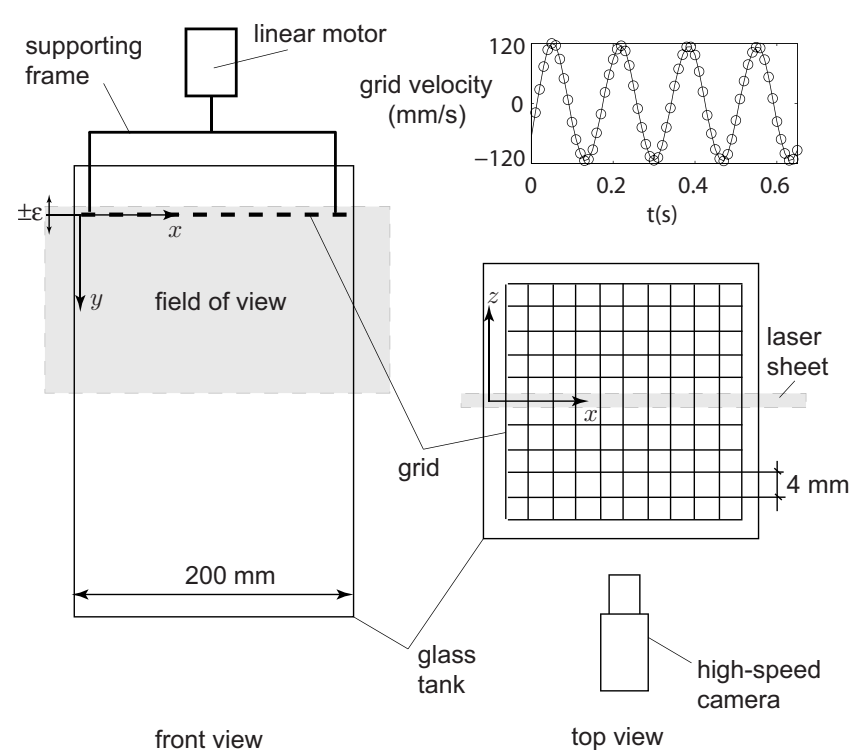

FIG. 1. Schematic of the oscillating grid-stirred tank experimental setup. A sample of the grid velocity in time is shown in the upper right corner.

front, i.e., $v_{e}=\beta v_{\text {rms }}$, where $\beta$ is the entrainment constant. Entrainment across the TNTI is characterized by the presence of a thin layer that is called "viscous superlayer" by Ref. 23, in analogy to the viscous sublayer in wall-bounded flows, through which vorticity "diffuses" from the turbulent to the irrotational side. Qualitatively the viscosity-related mechanism was predicted a long time ago, but only recently it became clear that the underlying physical process is the viscous interaction of vorticity and rate of strain that occurs on the small(est) turbulent scales. ${ }^{24-27}$ Reference 27 also shows that the local entrainment velocity $u_{a}$ across the interface is proportional to the Kolmogorov velocity $u_{\eta}$. We will show that polymer additives affect both, the overall propagation velocity and the local entrainment velocity. This also means that we measure the effect of polymers on the small and large scales of the flow.

We begin our analysis with a qualitative comparison of the interface shape and will then test whether the theoretical prediction $^{28}$ for the spreading of turbulence from a planar source in time also holds for polymer solutions. We are finally interested to measure the entrainment constant $\beta$. Section II describes the oscillating grid facility and the method that employs PIV and an interface detection procedure. In Sec. III experimental results on interface shape, propagation velocity, and entrainment rate are presented and discussed. In Sec. IV we conclude how polymers affect the role of large and small scales of the flow and what is the relation between them.

\section{EXPERIMENTAL METHOD}

The oscillating grid apparatus shown in Fig. 1 is the same as the one used in the previous investigations conducted by the authors. ${ }^{25-27}$ In Ref. 25 particle image velocimetry (PIV) and fluorescent dye visualization experiments were conducted to characterize the flow and describe the spreading of the turbulent flow region in time. The detailed description of the experimental apparatus and the PIV method are given in Ref. 25 and we summarize some basic parameters only. The glass tank $\left(20 \times 20 \times 30 \mathrm{~cm}^{3}\right)$ filled with water or aqueous dilute poly(ethylene oxide) solutions of Polyox WSR 301, MW=4 $\times 10^{6} \mathrm{~g} / \mathrm{mol}$ is fitted with a vertically oscillating grid (a fine woven screen of circular bars of $d=1 \mathrm{~mm}$, mesh size is $M=4 \mathrm{~mm}$ ). The motor, operated in a closed loop with feedback from a linear encoder, runs at a frequency of $f=6 \mathrm{~Hz}$ and a stroke of $S=8 \mathrm{~mm}$.

For the PIV measurements a high-speed camera (Photron Ultima APX) was used with a frame rate of 60 frames/s and an exposure time of $0.005 \mathrm{~s}$. The laser beam of a continuous $25 \mathrm{~W}$ Ar-ion laser was expanded through a cylindrical lens into a planar laser sheet less than $1 \mathrm{~mm}$ thick, passing through the midplane of the tank, as shown schematically in Fig. 1. The field of view of dimensions $200 \times 100 \mathrm{~mm}^{2} \mathrm{ex}-$ tends over the whole width of the tank. The camera recorded the light scattered by neutrally buoyant Polystyrene tracer particles with a diameter of $40 \mu \mathrm{m}$. The PIV images were processed with an interrogation windows of $16 \times 16$ pixels, $50 \%$ overlap, yielding approximately 8000 two-component velocity vectors per realization, denoted as $u$ and $v$ in $x$ (horizontal) and $y$ (vertical) directions, respectively.

Six runs each were performed for clear water flow and for aqueous dilute poly(ethylene oxide) solutions at two concentrations of 25 and 50 wppm. We detect the TNTI by using the method based on the out-of-plane vorticity component described in Ref. 25. For the sake of brevity we briefly describe only the main steps: (a) the out-of-plane vorticity component $\omega_{z}$ is estimated using the two-component, twodimensional velocity vector fields, as $\partial v / \partial x-\partial u / \partial y$, (b) the threshold $\omega_{z}^{*}$ is chosen above the noise level using a semiautomatic procedure, ${ }^{25}$ (c) at every $x$, we find the lowest position $y^{*}$ at which $\omega_{z} \leq \omega_{z}^{*}$. This location defines the instantaneous interface position, i.e., $\zeta(x, t)=y^{*}(x, t)$.

\section{RESULTS}

\section{A. Qualitative results: Modified shape of turbulent/nonturbulent interface}

Following Refs. 23 and 29 the TNTI can be represented in general as a convoluted surface $\zeta(x, z, t)$ or specifically in the case of a two-dimensional representation as a curve $\zeta(x, t)$, as sketched in Fig. 2(a). The mean depth of the turbulent region, $H(t)$, is estimated as $H(t)=\overline{\zeta(x, t)}$, where the overbar denotes the average over the horizontal dimension $x$.

We distinguish between the entrainment velocity $u_{a}$ and the propagation velocity $v_{e}$. The latter is the advancement of the mean position of the interface toward the nonturbulent region, $v_{e}=d H / d t$. This velocity is by similarity related to the velocity scale of energy-containing eddies of motion, the root mean square of the turbulent fluctuating velocity $v_{\text {rms }}$. As conjectured already ${ }^{23}$ and recently confirmed through direct measurements, ${ }^{27} u_{a}$ is of the order of the Kolmogorov velocity scale, $u_{\eta}=(\varepsilon \nu)^{1 / 4}$ ( $\varepsilon$ is the dissipation rate and $\nu$ is the kinematic viscosity). The two velocities are related through the interface geometry by the following relation: ${ }^{29}$

$$
v_{e}=u_{a}\left[\overline{1+(\nabla \zeta)^{2}}\right]^{1 / 2} \text {. }
$$


a)

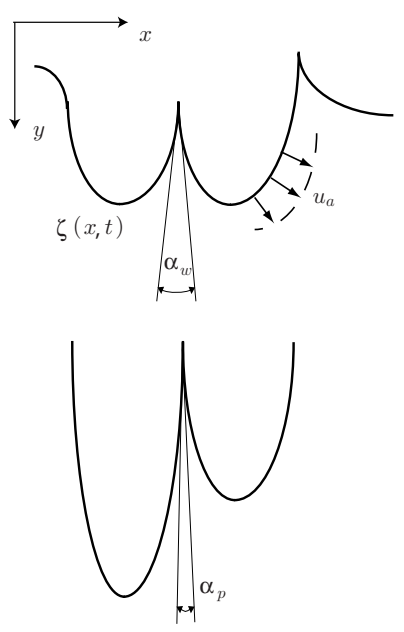

b)
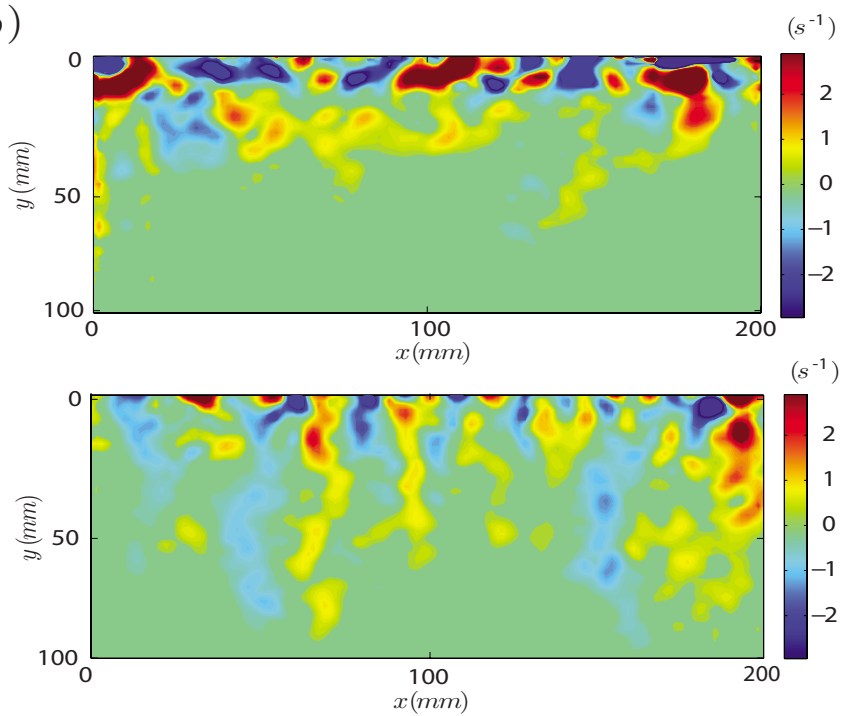

FIG. 2. (Color online) (a) Schematic description and definitions of the frame of reference and notations and (inset) typical shape of the interface for different ratios of $v_{\text {rms }} / u_{a}$, according to Ref. 29. (b) Interface shape of the turbulent front propagating under an oscillating grid. The upper panel is clear water and the lower one dilute polymer solution, respectively. Contours and colors represent instantaneous distributions of the out-of-plane component of vorticity, $\omega_{z}$.

The general problem is to find the evolution of $\zeta$ given initial conditions $\zeta\left(x, t_{0}\right)$. Reference 29 stated that the general case is "peculiarly intractable," but he could solve it for simpler cases, of which one is analogous to the present experiment (point 5.1 on page 109 of Ref. 29): the horizontal components of velocity are zero in the mean, and the vertical component is represented by the velocity scale $v_{\text {rms }}$. Reference 29 obtained the following relation, which describes the angle $\alpha$ formed by re-entrant zones of interfacial wedges, sketched in Fig. 2(a):

$$
\alpha=\sin ^{-1}\left(\frac{u_{a}}{2 v_{\mathrm{rms}}+u_{a}}\right) \text {. }
$$

From this relation it is clear that the smaller the ratio $u_{a} / v_{\mathrm{rms}}$ - the deeper the wedges and the higher their aspect ratio. Visual comparison for the shape of TNTI between water and polymer flow, shown in Fig. 2(b), qualitatively demonstrates that the aspect ratio of large scale interfacial wedges is increased by polymers. Unfortunately, the spatial resolution of our experiment is not sufficient to analyze the angle directly.

\section{B. Quantitative results: Propagation velocity and entrainment rate}

In this section we compare the propagation of the turbulent front in water and dilute polymer solutions. The first result in Fig. 3 shows the evolution in time of the depth of the turbulent layer $H(t)$. Three curves are plotted representing water, a $25 \mathrm{wppm}$ and a $50 \mathrm{wppm}$ polymer solution, respectively. Each curve is an average over six experimental runs, the error bars in the left (linear scale) panel or scatter (log-log scale) in the right panel represent the variance. In each run small spurious vertical velocity values due to the secondary flow typical for the rectangular tanks of small volume were subtracted before the analysis.

Reference 28 analyzed the propagation of a turbulent flow, driven by the planar energy source (e.g., vertically os-

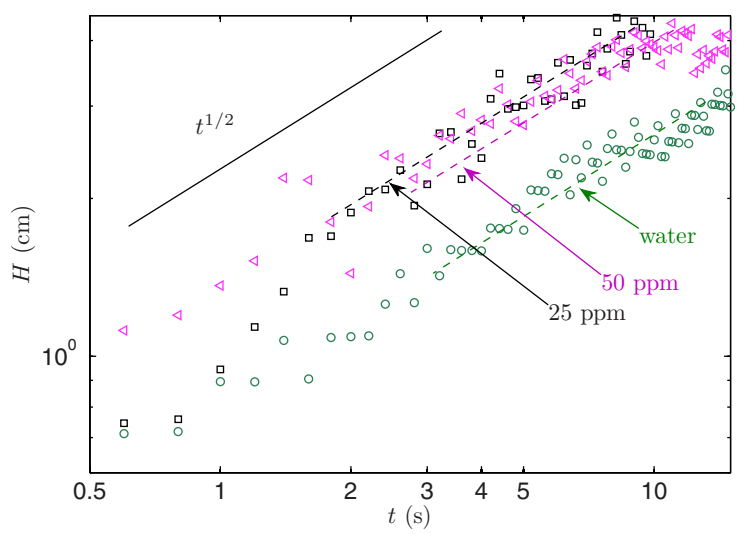

FIG. 3. (Color online) Depth of the turbulent layer, $H(t)$, in water and dilute polymer solutions of 25 and 50 ppm, shown as symbols in linear (left) and log-log (right) scales. The curves represent the best fit of the form $H=\sqrt{K t}$. Inset: the values of $K$ as a function of concentration. 

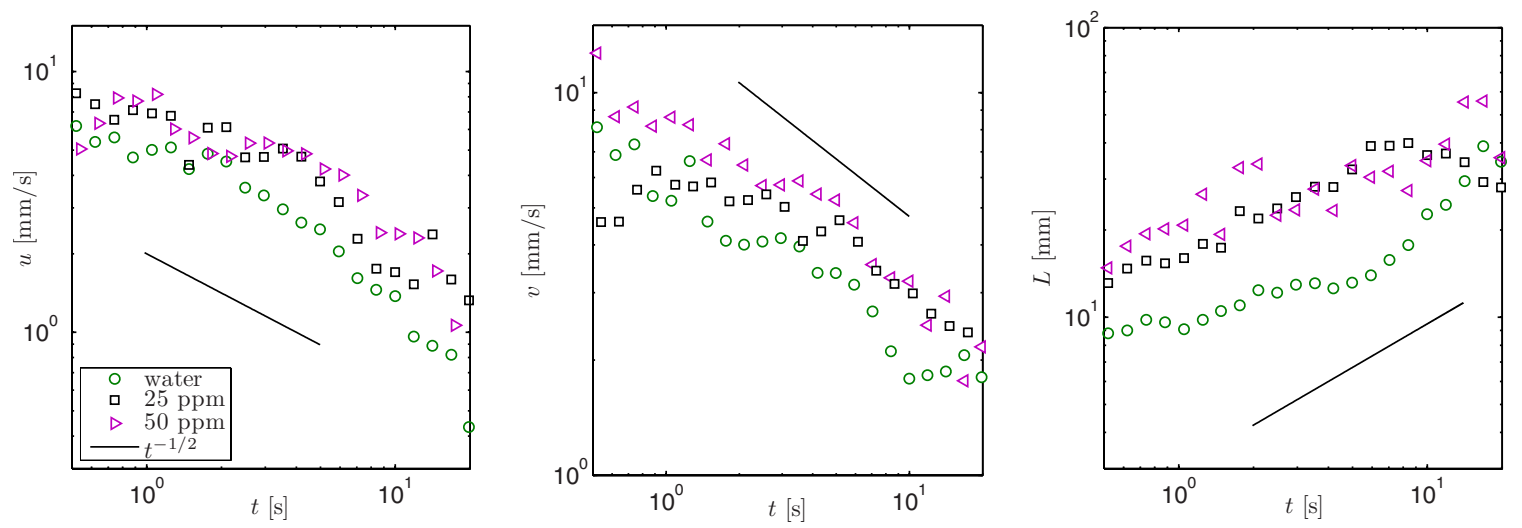

FIG. 4. (Color online) Horizontal (left), vertical (center), and length (right) scales estimate, measured at the TNTI. Solid lines indicate slopes $t^{ \pm 1 / 2}$.

cillating grid) in time and predicted $H=\sqrt{K}$. Reference 25 recently verified this prediction for clear water in our apparatus. In the present study we extend this analysis to dilute polymer solutions. From regression analysis using $H(t)$ $=K^{1 / 2} t^{n}$ or $\ln (H)=1 / 2 \ln (K)+n \ln (t)$ (Ref. 25) we obtained $n=0.47,0.52$, and $0.48 \pm 0.05$ for the three cases, respectively, and henceforth used $n=0.5$ for all cases.

The results obtained for the "grid action" are $K_{w}$ $=87 \pm 45, K_{25}=156 \pm 45$, and $K_{50}=144 \pm 45 \mathrm{~mm}^{2} / \mathrm{s}$ for water, 25 and $50 \mathrm{ppm}$, respectively. The values of $K$ as a function of concentration are plotted as an inset in Fig. 3. It is interesting that $K_{50}$ is less than $K_{25}$ and the trend seems nonmonotonic, however, the difference is small and lies within the experimental error. More experiments would be needed also for different concentrations to analyze this point in more detail and this is left open for future research. We notice that the TNTI propagates faster in dilute polymers solutions. It appears that the "efficiency" of the grid, $\gamma=K /\left(S^{2} f\right)$, increases for polymers: $\gamma_{w}=0.23,0.41$, and $0.38 \pm 0.1$ for water, 25 and $50 \mathrm{ppm}$, respectively. This translates into an increased turbulent kinetic energy in the polymer solution flows. Figure 4 demonstrates the change of the velocity scales measured in the proximity of the interface and the length scale estimate, based on the correlation analysis along the interface. With these results we are able to determine the changes in Reynolds number and the changes of the input energy. Taking the characteristic length scale $L$ of the turbulent flow to be proportional to the thickness of the turbulent layer ${ }^{28} H(t)$, or using the direct estimate based on the correlation analysis (Fig. 4), we note that $L$ increases by $30 \%$ in dilute polymer solutions. In addition, we measured an increase in the characteristic velocity scale, $v_{\text {rms }}$, of about $60 \%$, which is shown in Fig. 4. It follows that the Reynolds number $\operatorname{Re}=v_{\text {rms }} L / \nu$ is twice as large in the dilute polymer solution flow than in water. We remind that Polyox WSR301 is known to have approximately the same viscosity as water in dilute solutions. ${ }^{8}$

Finally we estimate the entrainment constant, $\beta$ $=v_{e} / v_{\text {rms. }}$. The propagation velocity of the TNTI is taken from the PIV measurements as $v_{e}=1 / 2 K^{1 / 2} t^{-1 / 2}$. For the estimate of $v_{\text {rms }}$, data points were used at $y=H(t)$ in turbulent flow regions only. It means that the points are from the subset of $\zeta(x, t)>H(t)$, where velocity fluctuations are due to turbulence. For water, 25 and $50 \mathrm{ppm}$ data we obtain $\beta_{w}$ $=0.77, \beta_{25}=0.68$, and $\beta_{50}=0.70 \pm 0.07$, respectively.

We note that the reduced entrainment rate in oscillation grid flow of drag-reducing fluids reminds of other free shear flows, for example, jets, mixing layers, and wakes. With polymers, these flows have been observed to develop significantly smaller spreading angles of the turbulent region. ${ }^{30}$

\section{DISCUSSION}

In the following we develop some considerations based on similarity principles and fractal theory to bring our results in context with other findings in literature. There is no proof that such arguments, valid for Newtonian fluids, remain applicable also with the presence of polymers, but the previous observation that the flow remains self-similar indicates that these assumptions are reasonable, at least for the range of parameters investigated herein. We can estimate the energy flux as $\varepsilon \propto v_{\text {rms }}^{3} / L$. In the case of clear water this energy flux is equal to dissipation through viscosity by the smallest scales of turbulence, $\varepsilon_{\nu}=2 \nu\left\langle s_{i j} s_{i j}\right\rangle\left(s_{i j}\right.$ is the fluctuating rate of strain tensor). When polymers are introduced, $\varepsilon$ is partly dissipated through the deformation of flexible polymer molecules $\varepsilon_{p}$, while only a fraction $\delta$ is dissipated through the viscous eddies and we write $\varepsilon_{\nu}=\delta \varepsilon$ or $\varepsilon_{p}=(1-\delta) \varepsilon$. Reference 31 suggested that it is likely that polymers intercept the energy flux at scales $r$, which are between the Lumley scale and the Kolmogorov scale, $r_{L}>r>\eta$, with $r_{L}=\sqrt{\epsilon_{T} \tau^{3}}$. This results in an unaffected inertial range, where the energy flux $\epsilon_{T}$ is constant, at low wave numbers and in a leakage at a higher wavenumber range $k>1 / r_{L}$, where the energy flux is progressively reduced by the dissipation of the polymers. Our results suggest that $r_{L}$ is not very sharp, since nominally in our experiment $r_{L} \leq \eta$ and De $\leq 1$ and still, we see a clear effect of the polymers in the bulk properties of our flow.

The entrainment flux can be expressed using a projected area $A_{L}$ or the area $A_{\eta}$ resolved up to the smallest length scales $^{26}$

$$
v_{e} A_{L}=u_{\eta} A_{\eta}
$$

With $\varepsilon \approx C v_{\text {rms }}^{3} / L$ the small scale quantities $u_{\eta}$ and $A_{\eta}$ can be expressed in terms of large scale quantities as 


$$
u_{\eta}=\nu^{1 / 4} \varepsilon^{1 / 4} \approx C^{1 / 4} v_{\mathrm{rms}}^{3 / 4} \nu^{1 / 4} L^{-1 / 4} .
$$

Reference 32 proposed the following relation based on fractal theory with $d=7 / 3$ :

$$
\begin{aligned}
A_{\eta} \approx A_{L}\left(\frac{\eta}{L}\right)^{2-d} & \approx A_{L} \frac{L^{1 / 3} \varepsilon^{1 / 12}}{\nu^{1 / 4}} \\
& \approx A_{L} L^{1 / 3} C^{1 / 12} v_{\mathrm{rms}}^{1 / 4} L^{-1 / 12} \nu^{-1 / 4}
\end{aligned}
$$

Substituting Eqs. (4) and (5) into Eq. (3) and invoking $v_{e}=\beta v_{\mathrm{rms}}$ and $C=\delta C_{*}$ we obtain

$$
\beta \approx \delta^{1 / 3} C_{*}^{1 / 3} .
$$

From Eq. (6) it is possible to determine $\delta$ via the measured values of $\beta$. In the water flow $\delta=1$ by definition and with $\beta_{w} \approx 0.77$ we obtain $C_{*} \approx 0.46$. Therefore, with $\beta_{p}=0.7$ we obtain $\delta_{p}=0.72$ implying that $72 \%$ of the energy input is dissipated by viscous eddies, while $28 \%$ is dissipated by the polymers. This value is consistent with our estimates of $\delta$ $=0.7$ in the same Deborah number range, obtained using another setup of eight counter-rotating disks. ${ }^{10,18}$

As it is widely accepted, the most important dimensionless number that characterizes the effects of polymers in turbulence is the Deborah number $\mathrm{De}=\tau_{R} / \tau_{\eta}$, the ratio of the polymer characteristic time scale to the Kolmogorov time scale. The relaxation time $\tau_{R}$ of the polymers can be estimated by using the models of Zimm or Carreau for Polyox WSR $301\left(4 \times 10^{6} \mathrm{~g} / \mathrm{mol}\right)$. We estimate the characteristic time scale to be $\tau_{R} \approx 0.001$ to $0.1 \mathrm{~s}$. Estimating $\tau_{\eta}$ to be of order $\mathcal{O}(0.1) \mathrm{s}$, our De is between 0.01 and 1 . We also estimate the Lumley scale, defined as $r_{L}=\sqrt{\varepsilon_{T} \tau_{p}^{3}}$, Ref. 33. Again, assuming that the relation $\varepsilon_{T} \propto u^{3} / L$ is valid for the order of magnitude estimate we obtain $r_{L} \approx\left(3 \times 10^{-4}\right)-(3$ $\left.\times 10^{-1}\right) \mathrm{mm}$, which is smaller or equal to the Kolmogorov length scale.

Figure 5 shows the plot of $\delta$ versus De in which we add our experimental estimate to the data from numerical and experimental studies. ${ }^{6,9,10,18}$ At $\mathrm{De}=0$ the trend line has to cross unity (clear water), and at large Deborah numbers we assume the relation to level off asymptotically. It opens an interesting question for future research whether there is a relation between this asymptotic trend and the maximum drag reduction asymptote known from wall-bounded turbulent flows. We do not show the numerical results ${ }^{5,33}$ in Fig. 5, because their configurations are different-either with respect to the forcing (former), or with respect to polymer concentration (latter). Reference 33 use Oldroyd-B-type models of dilute polymers and keep the energy content of the large scales constant. Reference 5 model discrete dumbbells and the feasible number density was at the time 1 dumbbell $/ \eta^{3}$. Both report higher values for $\delta$.

\section{SUMMARY AND CONCLUSIONS}

The propagation of the TNTI in grid-stirred turbulent flows was studied in water and dilute polyethylene oxide solutions by using PIV. Several effects of polymer additives on the large and small scale flow properties and the relation between them were singled out.

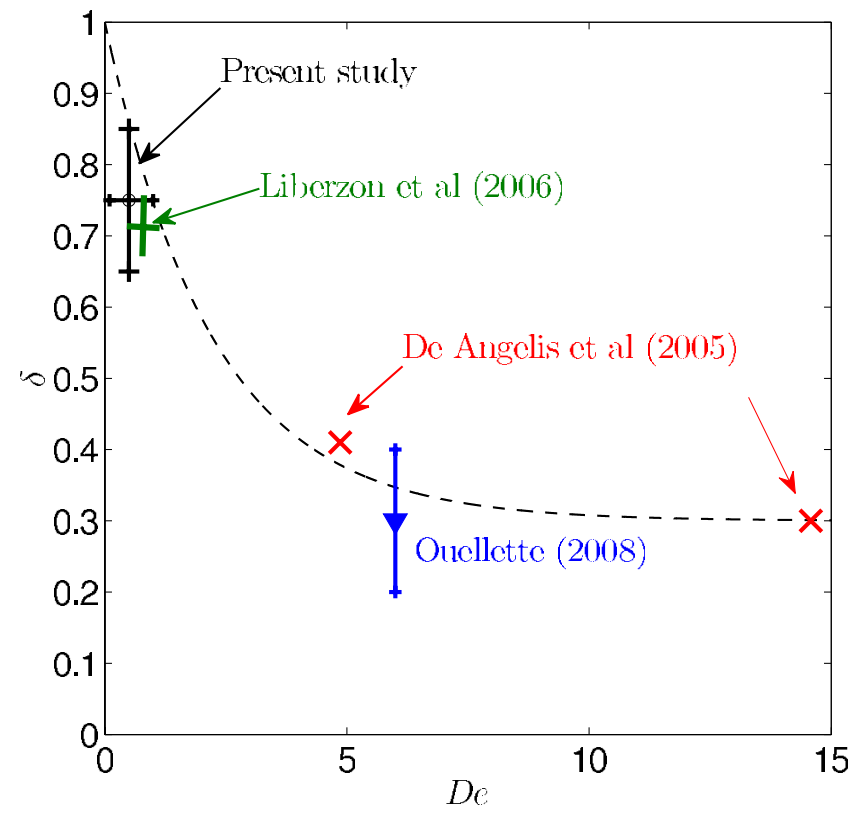

FIG. 5. (Color online) Dependence of $\delta=\varepsilon_{\nu} / \varepsilon_{i}$ on the Deborah number. $\times$ marks the numerical results (Ref. 6), a triangle marks the result of Ouellette et al. (Ref. 34; see also Ref. 9), + is for Ref. 18, and the circle denotes the present estimate. The error bars display the experimental uncertainty in the case of the present experiment and the variation of concentration in case of the experiment of Ref. 9, respectively.

The interface propagation in polymer solutions follows the same law $(\sqrt{K t})$ that was theoretically predicted and verified for clear water in previous experimental studies. However, we found that the turbulent front propagates faster in polymer solution than in water due to a higher grid action, $K_{p}>K_{w}$. The polymer flow thus extracts more power from the external forcing and changes the energy-containing scales. The integral velocity and length scales of the flow are observed to increase by about 60 and 30\%, respectively.

On a qualitative level we observe differences in the large scale shape of the TNTI: in agreement with predictions based on a simple model, ${ }^{29}$ the aspect ratio of interfacial wedges was observed to be increased by polymers. This effect is also reflected in changes in the structure and entrainment ability of the flow.

We found that polymers reduce the entrainment constant $\beta$. The measured values $\beta_{w}=0.8$ and $\beta_{p}=0.7$ for water and polymer are interpreted with a similarity analysis; we infer that in our experiment a fraction $\delta$ of the input energy of about $30 \%$ is dissipated by polymers, while the rest is dissipated by the smallest scales of turbulence. This is shown to be consistent with the dependence of $\delta$ on the Deborah number as obtained through a compilation of different results from literature. Despite the fact that the smallest scales of turbulence dissipate only a part of the input energy through viscosity, the values of total dissipation and $\mathrm{Re}$ are higher than the respective values for water. This alteration of the energy-containing scales reflects the somewhat surprising finding that polymers are responsible for an increased entrainment flux but at the same time lead to a decreased entrainment constant $\beta$.

Our work might be relevant for other applications of 
drag-reducing fluids, such as mixing layers, jets, and wakes, in which the entrainment rate was observed to decrease, along with larger, elongated scales of the flow. Undoubtedly, the presented study is limited in scope and experiments were performed in two dimensions only. In addition, the question of dependence on concentration remains open for a future study. A more detailed investigation using a threedimensional (3D) experimental method, such as 3D particle tracking velocimetry, would be beneficial, in addition to the thermal isolation of the system and direct measurements of the input power, similar to Ref. 8. This will allow to quantify the power dissipation and drag reduction effects. Nevertheless, the present work indicates that it is possible to estimate the polymer dissipation indirectly from the entrainment constant, without the need of difficult measurements of velocity gradients at the small scale level (i.e., dissipation). In fact, in the future we plan to estimate $\delta$ through measurements of $\beta$ for a variety of Re and De numbers and in different flows.

${ }^{1}$ B. A. Toms, "Some observation on the flow of linear polymer solutions through straight tubes at large Reynolds numbers," Proceedings of the First International Congress on Rheology, 1948, Vol. II, p. 135-141.

${ }^{2}$ A. Gyr and H. W. Bewersdorff, Drag Reduction of Turbulent Flows by Additives: Fluid Mechanics and its Applications (Kluwer, Dordrecht, 1995).

${ }^{3}$ R. H. Nadolink and W. W. Haigh, "Bibliography on skin friction reduction with polymers and other boundary-layer additives," Appl. Mech. Rev. 48, 351 (1995)

${ }^{4}$ C. D. Dimitropoulos, Y. Dubief, E. S. G. Shaqfeh, P. Moin, and S. K. Lele, "Direct numerical simulation of polymer-induced drag reduction in turbulent boundary layer flow," Phys. Fluids 17, 011705 (2005).

${ }^{5}$ T. Peters and J. Schumacher, "Two-way coupling of finitely extensible nonlinear elastic dumbbells with a turbulent shear flow," Phys. Fluids 19, 065109 (2007).

${ }^{6}$ E. De Angelis, C. Casciola, R. Benzi, and R. Piva, "Homogeneous isotropic turbulence in dilute polymers," J. Fluid Mech. 531, 1 (2005).

7 M. P. Tulin and J. Wu, "Additive effects on free turbulent flows," Phys. Fluids 20, S109 (1977).

${ }^{8}$ O. Cadot, D. Bonn, and S. Douady, "Turbulent drag reduction in a closed flow system: Boundary layer versus bulk effects," Phys. Fluids 10, 426 (1998).

${ }^{9}$ A. M. Crawford, N. Mordant, H. Xu, and E. Bodenschatz, "Fluid acceleration in the bulk of turbulent dilute polymer solutions," New J. Phys. 10, 123015 (2008).

${ }^{10}$ A. Liberzon, M. Guala, B. Lüthi, W. Kinzelbach, and A. Tsinober, "Turbulence in dilute polymer solutions," Phys. Fluids 17, 031707 (2005).

${ }^{11} \mathrm{G}$. E. Gadd, "Turbulence damping and drag reduction produced by certain additives in water," Nature (London) 206, 463 (1965).

${ }^{12}$ G. E. Gadd, "Effects of long-chain molecule additives in water on vortex streets," Nature (London) 211, 169 (1966).
${ }^{13}$ G. E. Gadd, "Reduction of turbulent friction in liquids by dissolved additives," Nature (London) 212, 874 (1966).

${ }^{14}$ C. A. Greated, "Effect of polymer additive on grid turbulence," Nature (London) 224, 1196 (1969).

${ }^{15}$ W. D. McComb, J. Allan, and C. A. Greated, "Effect of polymer additives on the small-scale structure of grid-generated turbulence," Phys. Fluids 20, 873 (1977).

${ }^{16}$ C. A. Friehe and W. H. Schwarz, "Grid-generated turbulence in dilute polymer solutions," J. Fluid Mech. 44, 173 (1970).

${ }^{17}$ E. van Doorn, C. M. White, and K. R. Sreenivasan, "The decay of grid turbulence in polymer and surfactant solutions," Phys. Fluids 11, 2387 (1999).

${ }^{18}$ A. Liberzon, M. Guala, W. Kinzelbach, and A. Tsinober, "On turbulent kinetic energy production and dissipation in dilute polymer solutions," Phys. Fluids 18, 125101 (2006).

${ }^{19} \mathrm{~J}$. Wu, "An experiment on turbulent diffusion in additive solutions," AIChE J. 17, 1408 (1971).

${ }^{20}$ I. P. D. De Silva and H. J. S. Fernando, "Oscillating grids as a source of nearly isotropic turbulence," Phys. Fluids 6, 2455 (1994).

${ }^{21}$ E. J. Hopfinger and J. A. Toly, "Spatially decaying turbulence and its relation to mixing across density interfaces," J. Fluid Mech. 78, 155 (1976).

${ }^{22}$ A. A. Townsend, The Structure of Turbulent Shear Flow (Cambridge University Press, Cambridge, 1976).

${ }^{23}$ S. Corrsin and A. L. Kistler, "Free-stream boundaries of turbulent flows," Technical Report No. TN-3133, NACA 1954; S. Corrsin and A. L. Kistler, "Free-stream boundaries of turbulent flows," Technical Report No. TR1244, NACA 1955.

${ }^{24}$ J. Westerweel, T. Hofmann, C. Fukushima, and J. Hunt, "The turbulent/ non-turbulent interface at the outer boundary of a self-similar turbulent jet," Exp. Fluids 33, 873 (2002).

${ }^{25}$ M. Holzner, A. Liberzon, M. Guala, A. Tsinober, and W. Kinzelbach, "Generalized detection of a turbulent front generated by an oscillating grid," Exp. Fluids 41, 711 (2006).

${ }^{26}$ M. Holzner, A. Liberzon, N. Nikitin, W. Kinzelbach, and A. Tsinober, "Small scale aspects of flows in proximity of the turbulent/non-turbulent interface," Phys. Fluids 19, 071702 (2007).

${ }^{27}$ M. Holzner, A. Liberzon, N. Nikitin, B. Lüthi, W. Kinzelbach, and A. Tsinober, "A Lagrangian investigation of the small scale features of turbulent entrainment through particle tracking and direct numerical simulation," J. Fluid Mech. 598, 465 (2008).

${ }^{28}$ R. R. Long, "Theory of turbulence in a homogeneous fluid induced by an oscillating grid," Phys. Fluids 21, 1887 (1978).

${ }^{29}$ O. M. Phillips, "The entrainment interface," J. Fluid Mech. 51, 97 (1972).

${ }^{30} \mathrm{~J}$. W. Hoyt, "Effect of additives on fluid friction," ASME J. Basic Eng. 94, 258 (1972)

${ }^{31}$ C. M. Casciola and E. De Angelis, "Energy transfer in turbulent polymer solutions," J. Fluid Mech. 581, 419 (2007).

${ }^{32}$ K. R. Sreenivasan, "Fractals and multifractals in fluid turbulence," Annu. Rev. Fluid Mech. 23, 539 (1991).

${ }^{33}$ S. Berti, A. Bistagnino, G. Boffetta, A. Celani, and S. Musacchio, "Smallscale statistics of viscoelastic turbulence," Europhys. Lett. 76, 63 (2006).

${ }^{34} \mathrm{~N}$. T. Ouellette, H. Xu, and E. Bodenschatz, "Bulk turbulence in dilute polymer solutions," J. Fluid Mech. (in press) 